\title{
Charmonium propagation through a dense medium
}

\author{
B.Z. Kopeliovich ${ }^{1}$, a , I.K. Potashnikova ${ }^{1}$, Iván Schmidt ${ }^{1}$, and M. Siddikov ${ }^{1}$ \\ ${ }^{1}$ Departamento de Física, Universidad Técnica Federico Santa María; and \\ Centro Científico-Tecnológico de Valparaíso; Avda. España 1680, Valparaíso, Chile
}

\begin{abstract}
Attenuation of a colourless $\bar{c} c$ dipole propagating with a large momentum through a hot medium originates from two sources, Debye screening (melting), and inelastic collisions with surrounding scattering centres (absorption). The former never terminates completely production of a bound charmonium in heavy ion collisions, even at very high temperatures. The latter, is controlled my the magnitude of the dipole cross section, related to the transport coefficient, which is the rate of transverse momentum broadening in the medium. A novel procedure of Lorentz boosting of the Schrödinger equation is developed, which allows to calculate the charmonium survival probability employing the path-integral technique, incorporating both melting and absorption. A novel mechanism of charmonium regeneration in a dense medium is proposed.
\end{abstract}

\section{Introduction}

Charmonium attenuation in a hot matter has been traditionally considered to be sensitive to the medium temperature. If the Debye screening radius becomes smaller than the charmonium, the bound level disappears and the charmonium dissociates into an unbound $\bar{c} c$ pair. However, no clear signal of such an effect has been reported so far, after two decades of experimenting.

The effects of melting has been usually calculated assuming charmonium to be at rest relative to the medium, while its mean momentum squared measured within the collisions energy interval of the $\mathrm{LHC}$, ranges between 7 and $10 \mathrm{GeV}^{2}$ [1]. The charmonium was assumed to be either completely terminated [2], once the bound level is eliminated by screening, or the decay of the bound state was described by introducing an effective imaginary part of the potential, giving rise to a decay width [3].

Another source of charmonium break-up caused by colour-exchange interactions with the surrounding matter [4]) has been missed in previous calculations. At the same time, this mechanism has been known for hadrons propagating through nuclear matter since long time ago within the Glauber model.

Apparently, absorption becomes even stronger in a medium,which is much denser than nuclei. However, the dipole cross section of inelastic interaction with a dense medium is unknown, and hardly can be fixed by other data. The important observation, made in [5], is a relation between the imaginary part of the potential and the transport coefficient, which is related to the medium temperature (see below).

Propagation of colour dipoles through a medium was proposed in [6, 7] to describe with the path-integral technique, which sums-up all possible trajectories of propagation in the medium .

\footnotetext{
a e-mail: boris.kopeliovich@usm.cl
} 


\section{Absorption}

Within the Glauber model [8] a high-energy hadron propagating through nuclear matter attenuates exponentially, with the rate proportional to the nuclear density and the inelastic hadron-nucleon cross section. Colour transparency makes the medium less opaque, an a dipole attenuates as inverse power of the path length, rather than exponentially [9]. The source of attenuation, the possibility of inelastic interactions of the hadron, which result from colour exchanges in the cross channel. Survival of the dipole corresponds to the probability for the hadron to avoid colour-exchange collisions, i.e. to remain colourless. This mechanism of attenuation is quite different from colour screening in a medium considered in the next section.

It is convenient to relate the rate of inelastic collisions in the nucleus, $\sigma_{\text {in }} \rho_{A}(l)$ with the transport coefficient $\hat{q}$, which is the rate of broadening of the transverse momentum $k_{T}$ of a parton propagating through the medium [10],

$$
\hat{q}(l) \equiv \frac{\partial \Delta k_{T}^{2}}{\partial l}
$$

Calculated within the framework of the dipole approach [9], the broadening rate was found to be[11],

$$
\hat{q}(l)=2 C \rho_{A}(l)
$$

where

$$
C=\left.\frac{1}{2} \vec{\nabla}_{r_{T}}^{2} \sigma_{\bar{q} q}\left(r_{T}\right)\right|_{r_{T}=0},
$$

and $\sigma_{\bar{q} q}\left(r_{T}\right)$ is the universal cross section dipole-proton cross section [9]. Besides the transverse $\bar{q} q$ separation $r_{T}$, it also implicitly depends on the dipole energy [12].

Employing the small- $r_{T}$ approximation, $\sigma_{\bar{q} q}\left(r_{T}\right) \approx C r_{T}^{2}$, we can represent the survival probability as,

$$
S^{2}\left(l_{1}, l_{2}\right)=\left\langle\exp \left[-\frac{r_{T}^{2}}{2} \int_{l_{1}}^{l_{2}} d l \hat{q}(l)\right]\right\rangle
$$

Here instead of averaging the dipole cross section in the exponent, as is done in the Glauber approximation, the whole exponential is averaged. This way the Gribov corrections $[9,13]$ are included to all orders (except gluon shadowing, which comes from higher Fock components [14]). This expression allows a direct generalization for absorption in a dense quark-gluon medium, which is usually characterized with a transport coefficient $\hat{q}$.

In Eq. (4) the dipole transverse separation $r_{T}$ is assumed to be "frozen" by Lorentz time dilation, i.e. to remain unchanged during the time of propagation through the medium, which requires high dipole energies. The path integral technique avoids this approximation and describes the propagation of a "breathing" dipole.

A $\bar{c} c$ pair produced in the c.m. frame of the colliding nuclei with a small initial separation $r_{0} \sim 1 / \sqrt{4 m_{c}^{2}+p_{T}^{2}}$ propagates through the medium with momentum $p_{\psi} \equiv p_{T}$. In such a kinematics of eaqual intrinsic and total momenta the initial dipole size can never be "frozen" by Lorentz time dilation.

A rigorous quantum-mechanical description of the propagation of a colourless $\bar{c} c$ can be performed with the path-integral method $[6,15]$. One has to sum up all possible trajectories of propagation of quark and antiquark in order to incorporate the effects of fluctuation of the dipole separation. In the light-cone variables the Green function of the dipole propagating in a medium satisfies the 2-dimensional Schrödinger-type equation $[6,15]$,

$$
i \frac{\partial}{\partial l} G\left(l, \vec{r}_{T} ; 0, \vec{r}_{0}\right)=\left[\frac{m_{c}^{2}-\Delta_{r_{T}}}{2 E_{\psi} \alpha(1-\alpha)}+U_{\bar{c} c}\left(r_{T}, l\right)\right] G\left(l, \vec{r}_{T} ; 0, \vec{r}_{0}\right),
$$


Here $\alpha$ and $1-\alpha$ are the fractional light-cone momenta of $c$ and $\bar{c} ; l$ is the longitudinal coordinate along the trajectory of the $\bar{c} c$ dipole in the rest frame of the medium. The dipole evolves its transverse separation from the initial value $\vec{r}_{0}$ at $l=0$ up to $\vec{r}_{T}$ after propagating a distance $l$. To avoid possible confusions we remind that $r_{T}$ is transverse relative to the charmonium trajectory, which itself is orthogonal to the nuclear collision axis.

The real part of the effective potential $\operatorname{Re} U\left(r_{T}\right)=V\left(r_{T}\right)$ is responsible for $\bar{c} c$ binding, while the imaginary part is responsible for interaction of the dipole and the medium,

$$
\operatorname{Im} U_{\bar{c} c}\left(r_{T}, l\right)=-\frac{v_{\psi}}{4} \hat{q}(l) r_{T}^{2},
$$

where $v_{\psi}=p_{\psi} / E_{\psi}$ is the dipole velocity relative to the medium, $E_{\psi}=\sqrt{p_{\psi}^{2}+4 m_{c}^{2}}$ is its energy.

The survival probability amplitude of a charmonium produced inside a hot medium is given by the convolution of the Green function with the initial and final distribution amplitudes,

$$
|S|^{2}=\left|\frac{\int d^{2} r_{1} d^{2} r_{2} \Psi_{f}^{\dagger}\left(r_{2}\right) G\left(L, \overrightarrow{r_{2}} ; 0, \overrightarrow{r_{1}}\right) \Psi_{i n}\left(r_{1}\right)}{\int d^{2} r \Psi_{f}^{\dagger}(r) \Psi_{i n}(r)}\right|^{2}
$$

The charmonium wave function $\Psi_{f}(r, \alpha)$ is dominated by equal sharing of the light-come momentum, $\alpha \approx 1 / 2$ (see more below).

\section{Debye screening}

\subsection{Maximal screening}

Intuitively is clear that if the binding potential is weakened (for whatever reason), or even completely eliminated, for a short time interval $\Delta t \ll 1 / \omega$ in the charmonium rest frame, the bound state will not be strongly disturbed, because the quarks will have no time to move away from their orbits. In the medium rest frame the formation time of the wave function is much longer than the propagation time in the medium, $t_{f} \gg R_{A} / v_{\psi}$. Thus, the Debye screening effect is expected to vanish for charmonia produced in heavy ion collisions with large $p_{T}=p_{\psi}$.

At this point we want to demonstrate on an exaggerated example of a binding potential, completely vanishing inside the medium (infinitely high temperature), that the survival probability of the dipole remains finite. Let us assume the following coordinate dependence of the potential (no time dependence),

$$
\operatorname{Re} U(r)=\left\{\begin{array}{cc}
\operatorname{Re} U(r)=0 & \text { inside the medium; } \\
\operatorname{Re} U(r)=\operatorname{Re} U_{v a c}(r) & \text { outside the medium. }
\end{array}\right.
$$

To clear up this example we simplify the scenario avoiding other sources of attenuation and inessential technical complications. In particular, we eliminate the absorptive imaginary part of the potential, Eq. (6); keep the Debye screening maximally strong along the whole path length $L$ of the $\bar{c} c$ in the medium; and use the oscillator form of the real part of the potential outside the medium. Correspondingly both the initial and final wave functions in (7) have the Gaussian form, $\Psi(r)=(\lambda / \sqrt{\pi}) \exp \left(-\lambda^{2} r^{2} / 2\right)$ with $\lambda^{2}=m_{c}^{2}+p_{\psi}^{2} / 4$ and $\lambda^{2}=m_{c} \omega / 2$ for initial and final size distribution functions respectively. Then the equation (5) can be solved analytically, and the suppression factor Eq. (7) takes the form [6],

$$
|S(L)|^{2}=\frac{m_{c}^{2} p_{\psi}}{16 \pi^{2} L}\left(1+\frac{\omega}{2 m_{c}}\right) \frac{8 \pi^{2}}{m_{c}^{2}}\left[\omega^{2} m_{c}^{2}+\frac{p_{\psi}^{2}}{4 L^{2}}\left(1+\frac{\omega}{2 m_{c}}\right)^{2}\right]^{-1 / 2},
$$


The resulting suppression factor $|S(L)|^{2}$ is plotted in Fig. 1 as function of $J / \psi$ momentum, and for different path lengths $L=1,3$, and $5 \mathrm{fm}$. Although in this exaggerating case the survival probability

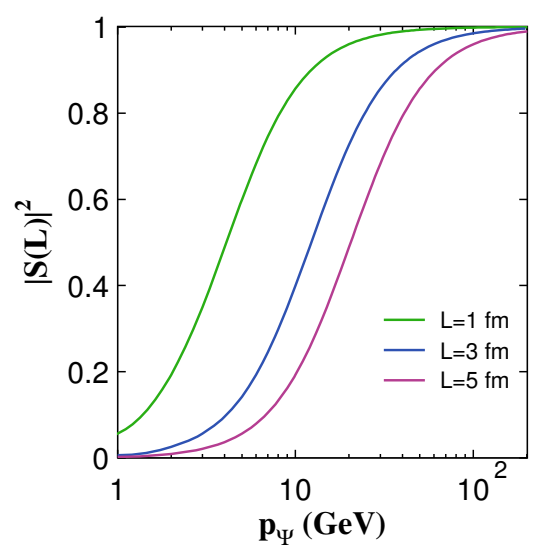

Figure 1. The attenuation factor $|S(L)|^{2}$ for $J / \psi$ produced with momentum $p_{\psi} \equiv p_{T}$ off a hot medium. The Debye screening in the medium is maximal, completely eliminating the $\bar{c} c$ binding potential during in-medium propagation over the path length $L$,

vanishes towards $p_{\psi}=p_{T}=0$, in reality (see below) even at small momenta $p_{\psi}$ the survival probability is fairly large because of the rapid decrease of temperature in expanding plasma and restoration of the binding potential. In another limit of high momentum the survival probability approaches unity because the expansion of the initially small dipole size is slowed down by Lorentz time dilation.

\subsection{Boost-invariant Schrödinger equation}

Sveral realistic parametrizations are currently available, which describe well the charmonium spectrum (see in [16]). However, such 3-dimensional potentials are appropriate only for a charmonium at rest, while the mean transverse momentum squared of $J / \psi$ produced in heavy ion collisions at LHC varies from 7 to $10 \mathrm{GeV}^{2}$ [1]. The same problem concerns the Debye screening effects, which are known from the lattice simulations [17-19] only in the charmonium rest frame.

In the large- $m_{c}$ limit the intrinsic motion of a system of heavy quarks is non-relativistic [20] and the Fock space of charmonium is dominated by the lowest $\bar{c} c$ state, whose dynamics can be described in terms of an effective potential. In this limit, the binding energy is small, so the difference of momenta carried by the quark and antiquark is also small, [21],

$$
\left\langle\lambda^{2}\right\rangle \equiv\left\langle(\alpha-1 / 2)^{2}\right\rangle=\frac{\left\langle p_{L}^{2}\right\rangle}{4 m_{c}^{2}}=\frac{1}{4}\left\langle v_{L}^{2}\right\rangle,
$$

where $\alpha$ is the quark fractional light-cone momentum; $p_{L}$ and $v_{L}$ are the longitudinal momentum and velocity of the charm quark in the rest frame of the charmonium. The mean value in (9), evaluated with realistic potentials, is rather small, $\left\langle\lambda^{2}\right\rangle=0.017$.

Here we present a brief description of the transformation procedure based on smallness of $\left\langle\lambda^{2}\right\rangle$ [22]. We assume that in the $\bar{c} c$ rest frame $V(r)$ is the interaction potential and its Fourier image $\tilde{V}(\vec{k})$ corresponds to the propagator of a hypothetical vector particle exchange in $t$-channel. The 


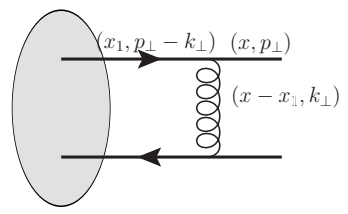

Figure 2. Kinematic variables for the Bethe-Salpeter bound state diagram.

Bethe-Salpeter equation for the eigenvalues is shown schematically in the Fig. 2 Making expansion over the small parameter $\lambda$, Eq. (9), we arrive at the equation of motion,

$$
p_{\psi}^{-} \phi\left(\alpha, r_{\perp}\right)=\frac{-\Delta_{\perp}+m_{c}^{2}}{2 p_{\psi}^{+} \alpha(1-\alpha)} \phi\left(\alpha, r_{\perp}\right)+\hat{V} \phi\left(\alpha, r_{\perp}\right),
$$

where $p_{\psi}^{ \pm}$are the components of the charmonium light-cone momentum. The interaction term here reads,

$$
\hat{V} \phi\left(\alpha, r_{\perp}\right)=\int_{0}^{1} d \beta K\left(\alpha, \beta, r_{\perp}\right) \phi\left(\beta, r_{\perp}\right),
$$

with the kernel $K\left(\alpha, \beta, r_{\perp}\right)$ given by

$$
\begin{aligned}
K\left(\alpha, \beta, r_{\perp}\right) \approx & \frac{1}{\pi} \frac{M_{\psi}}{p_{\psi}^{+}} \int_{r_{\perp}}^{\infty} d r r V(r) \frac{\cos \left(2 m_{c}|\alpha-\beta| \sqrt{r^{2}-r_{\perp}^{2}}\right)}{\sqrt{r^{2}-r_{\perp}^{2}}} \\
& =\frac{1}{2 \pi} \frac{M_{\psi}}{p_{\psi}^{+}} \int_{0}^{\infty} d z V\left(\sqrt{r_{\perp}^{2}+z^{2}}\right) e^{2 i m_{c}(\alpha-\beta) z}
\end{aligned}
$$

where $M_{\psi}$ is the charmonium mass. Since the kernel (11) depends only on the difference between the fractional light-cone momenta $\alpha-\beta$, it is convenient to work with a Fourier transform $\tilde{\phi}$ defined as

$$
\tilde{\phi}\left(z^{-}, r_{\perp}\right)=\int_{0}^{1} \frac{d \alpha}{2 \pi} \phi\left(\alpha, r_{\perp}\right) e^{2 i m\left(\alpha-\frac{1}{2}\right) z^{-}}
$$

where the variable $z^{-}$is the minus-component of the light-cone separation between quarks. Expanding (11) over a small parameter $\lambda$ and replacing $\lambda \Rightarrow i \partial z^{-} / 2 m$ in coordinate space, we get the final result for the Schrödinger equation [22],

$$
\frac{M_{\psi}^{2}}{2 p_{\psi}^{+}} \tilde{\phi}\left(z^{-}, r_{\perp}\right)=\frac{-\Delta_{\perp}-\partial_{z^{-}}^{2}+m_{c}^{2}}{p_{\psi}^{+} / 2} \tilde{\phi}\left(z^{-}, r_{\perp}\right)+\frac{M_{\psi}}{p_{\psi}^{+}} V\left(\sqrt{r_{\perp}^{2}+\left(z^{-}\right)^{2}}\right) \tilde{\phi}\left(z^{-}, r_{\perp}\right),
$$

which is explicitly invariant under Lorentz boosts. If we substitute now $M_{\psi} \approx 2 m_{c}+\epsilon_{\psi}$, where $\epsilon_{\psi}$ is the binding energy of the charmonium, in the charmonium rest frame in the leading order in $\epsilon_{\psi}$, we obtain the usual nonrelivistic Schrödinger equation. 
Now we can replace Eq. (5), in which the longitudinal motion of quarks was frozen, by an equation with the kinetic and potential terms, which describe the 3-dimentional motion of the quarks,

$$
\left[i \frac{\partial}{\partial z^{+}}+2 \frac{\Delta_{r_{T}}+\left(\partial / \partial z^{-}\right)^{2}-m_{c}^{2}}{p_{\psi}^{+}}-U_{\bar{c} c}\left(z^{-}, \vec{r}_{T}\right)\right] G\left(z^{+}, z^{-}, \vec{r}_{T} ; z_{1}^{+}, z_{1}^{-}, \vec{r}_{1 T}\right)=0 .
$$

Here the real part of the boosted potential is related to the rest frame binding potential $V(r)$ as, $\operatorname{Re} U\left(z^{-}, \vec{r}_{T}\right)=V\left(r=\sqrt{r_{T}^{2}+\left(z^{-}\right)^{2}}\right)$.

The real and imaginary, Eq. (6), parts of the potential are related to different parameters characterising the medium, the temperature and transport coefficient, respectively. At large $T \gg T_{c}$, where $T_{c}$ is the critical temperature, this relation is simple, $\hat{q} \approx 3.6 T^{3}$, but at lower temperatures $T \lesssim T_{c}$, it is is more complicated and is parametrized in [23].

\section{Results}

\subsection{Suppression of $J / \psi$}

Now we are in a position to solve numerically Eq. (14) for the Green function describing the propagation of a colourless $\bar{c} c$ pair through a hot medium, employing realistic potentials and screening corrections. As a model for the biding potential, we employ the Cornell form of the potential [24]. For medium screening effects we rely on the popular approach, which is based on the Debye-Hückel theory and the lattice results, parameterized in the analytic form proposed in [25],

$$
V_{\bar{c} c}(r, T)=\frac{\sigma}{\mu(T)}\left(1-e^{-\mu(T) r}\right)-\frac{\alpha}{r} e^{-\mu(T) r},
$$

where

$$
\mu(T)=g(T) T \sqrt{\frac{N_{c}}{3}+\frac{N_{f}}{6}} ; \quad g^{2}(T)=\frac{24 \pi^{2}}{33 \ln \left(19 T / \Lambda_{\overline{M S}}\right)} ;
$$

$\sigma=1 \mathrm{GeV} / \mathrm{fm}$ is the string tension; and the parameter $\alpha \approx 0.471$. The bound states in such a potential are terminated at high temperatures, when the Debye radius $r_{D}=1 / \mu(T)$ becomes smaller than the mean radius of the charmonium. We use this bound state potential in the real part of the boosted potential of Eq. (14).

The imaginary part of the boosted potential, Eq. (6) is controlled by the transport coefficient $\hat{q}$, varying with coordinates and time, for which we employ the popular model [23],

$$
\hat{q}(t, \vec{b}, \vec{\tau})=\frac{q_{0} t_{0}}{t} \frac{n_{\text {part }}(\vec{b}, \vec{\tau})}{n_{\text {part }}(0,0)} \Theta\left(t-t_{0}\right)
$$

Here $\vec{b}$ and $\vec{\tau}$ are the impact parameter of the collision and the point, where the medium is observed, respectively. We fix $t_{0}=1 \mathrm{fm}$. The maximal value of $\hat{q}$ at $\vec{b}=\vec{s}=0$ and $t=t_{0}$ is treated as a free parameter, to be adjusted to data.

The survival probability factor $S^{2}(b)$ of a charmonium produced in a collision of nuclei $A$ and $B$ with relative impact parameter $b$ reads (compare with (7)),

$$
\begin{aligned}
|S(b)|^{2} & =\int_{0}^{2 \pi} \frac{d \phi}{2 \pi} \int \frac{d^{2} s T_{A}(\vec{s}) T_{B}(\vec{b}-\vec{s})}{T_{A B}(b)} \\
& \times\left|\frac{\int d^{2} r_{1} d^{2} r_{2} d z_{1}^{-} d z_{2}^{-} \Psi_{f}^{\dagger}\left(\vec{r}_{2}, z_{2}^{-}\right) G\left(\infty, \vec{r}_{2}, z_{2}^{-} ; 0, \vec{r}_{1}, z_{1}^{-}\right) \Psi_{i n}\left(\vec{r}_{1}, z_{1}^{-}\right)}{\int d^{2} r d z^{-} \Psi_{f}^{\dagger}\left(\vec{r}, z^{-}\right) \Psi_{i n}\left(\vec{r}, z^{-}\right)}\right|^{2},
\end{aligned}
$$


where $\phi$ is the azimuthal angle between this trajectory and the scattering plane (vector $\vec{b}$ ).

The initial distribution function in (18) is assumed to have a Gaussian form, $\Psi_{i n}\left(\vec{r}_{T}, z^{-}\right)=$ $(a / \sqrt{\pi}) e^{-a^{2} r^{2} / 2}$, with $r=\sqrt{r_{T}^{2}+\left(z^{-}\right)^{2}}$, and $a \sim 1 / \sqrt{m_{c}^{2}+p_{T}^{2}}$. Since the initial expansion of a small dipole is very fast, the original separation is not important. The Green function $G\left(\infty, \vec{r}_{2}, z_{2}^{-} ; 0, \vec{r}_{1}, z_{1}^{-}\right)$, describing the dipole evolution, and the final wave function $\Psi_{f}\left(\vec{r}_{2}, z_{2}^{-}\right)$, are the solutions of Eqs. (13) and (14) respectively, with the same boosted potential.

To see the net melting effect we solve Eq. (14) fixing $\operatorname{Im} U=0$. The results for $|S(b=0)|^{2}$, calculated with Eq. (18) for a central lead-lead collision, are plotted in Fig. 3(left) for different trial values of the parameter $q_{0}=0.5,1,2$ and $4 \mathrm{GeV}^{2} / \mathrm{fm}$.
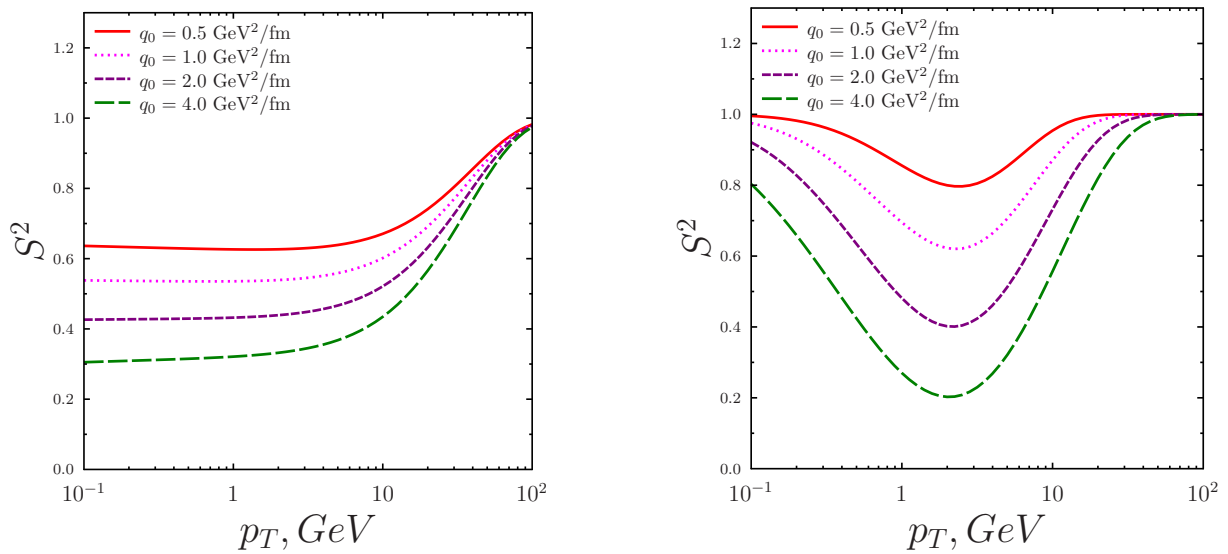

Figure 3. Left: The FSI suppression factor calculated with Eq. (18) for $J / \psi$ produced with momentum $p_{\psi} \equiv p_{T}$ in central lead-lead collisions. The absorption effects are eliminated by fixing $\operatorname{Im} U=0$, in order to single out the net effect of Debye screening. The curves from top to bottom are calculated with $q_{0}=0.5,1,2$ and $4 \mathrm{GeV}^{2} / \mathrm{fm}$. Right: The net effect of absorption, i.e. with eliminated Debye screening.

Notice that similar to Fig. 1 demonstrating the maximal melting effect, the survival probability $|S|^{2}$ rises with $p_{\psi}$ and saturates at unity. On the contrary, the behaviour at small $p_{\psi} \rightarrow 0$ in Fig. 3(left) is different from the case of maximal melting effect, because the transport coefficient Eq. (17) is finite and is falling with time as $1 / t$.

The other extreme is to eliminate the melting effect by fixing in (15) $T=0$, but include absorption introducing the imaginary part of the potential according to Eq. (6). In this way one can see the net effect of absorption. The results, using Eq. (18), are plotted in Fig. 3(right) for different values of $q_{0}$. Again, the survival probability approaches unity at high $p_{\psi}=p_{T}$ due to colour transparency.

The full results incorporating both effects of melting and absorption cannot be simply deduced from the above two extreme cases of net melting or net absorption effects, because they strongly correlate. Solving Eq. (14) with a potential containing the real and imaginary parts, and averaging over impact parameters with Eq. (18) we arrive at the final results for the suppression factor $|S|^{2}$ plotted in Fig. 4(left).

Eventually, for comparison, we plot all three versions presented in Figs. 3-4(left) on one plot Fig. 4(right) for the selected value of $q_{0}=2 \mathrm{GeV} / \mathrm{fm}$, extracted from the analysis [26] of data for high- $p_{T}$ hadron suppression in lead-lead collisions at LHC. We see that the magnitude of suppression caused by the two mechanisms is similar at transverse $J / \psi$ momenta around the mean value. The total suppression effect is maximal at $p \sim\left\langle p_{\psi}\right\rangle$ and is rather strong, about $|S|^{2} \approx 0.2$. 

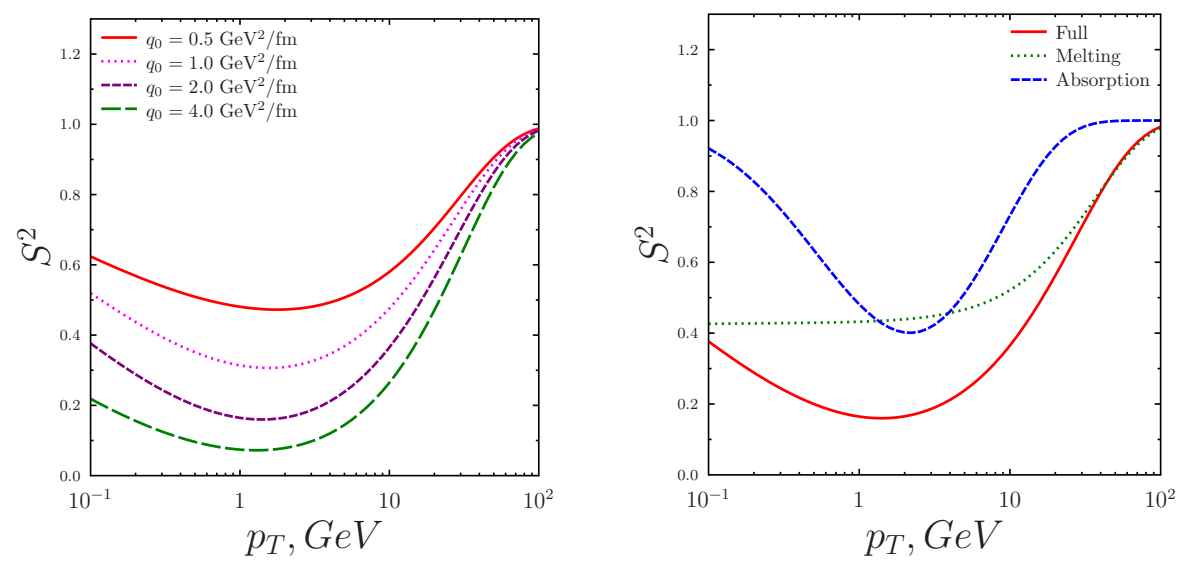

Figure 4. Left: Same as in Fig. 3, but including both, screening and absorption effects. Right: The curves from top to bottom show the attenuation factor $|S(L)|^{2}$ vs $J / \psi$ momentum for either pure absorption, or pure melting, and for both effects included, respectively. The calculations are performed for central lead-lead collisions with $q_{0}=2 \mathrm{GeV}^{2} / \mathrm{fm}$.

\subsection{Suppression of $\psi(2 S)$}

The first radial excitation $\psi(2 S)$ is known to have the mean radius squared about twice as big as $J / \psi$. Correspondingly, a stronger melting effect has been always expected for $\psi(2 S)$, as well as the effect of absorption, The suppression factor $|S|^{2}$ is given by the same Eq. (18), but the final wave function $\Psi_{f}$ is a solution of Eq. (12) for the radial excitation $\psi(2 S)$.

The results of calculations with $q_{0}=2 \mathrm{GeV}^{2} / \mathrm{fm}$ are shown in Fig. 5(left) for the suppression factors for net melting (dotted), net absorption (dashed) and full effect (solid). As expected, suppression depicted in Fig. 5 is considerably stronger compared with $J / \psi$. This is confirmed by the $\psi(2 S)$ to $J / \psi$ ratio plotted in Fig. 5(right), which is indeed mostly below unity. However, this ratio also demon-
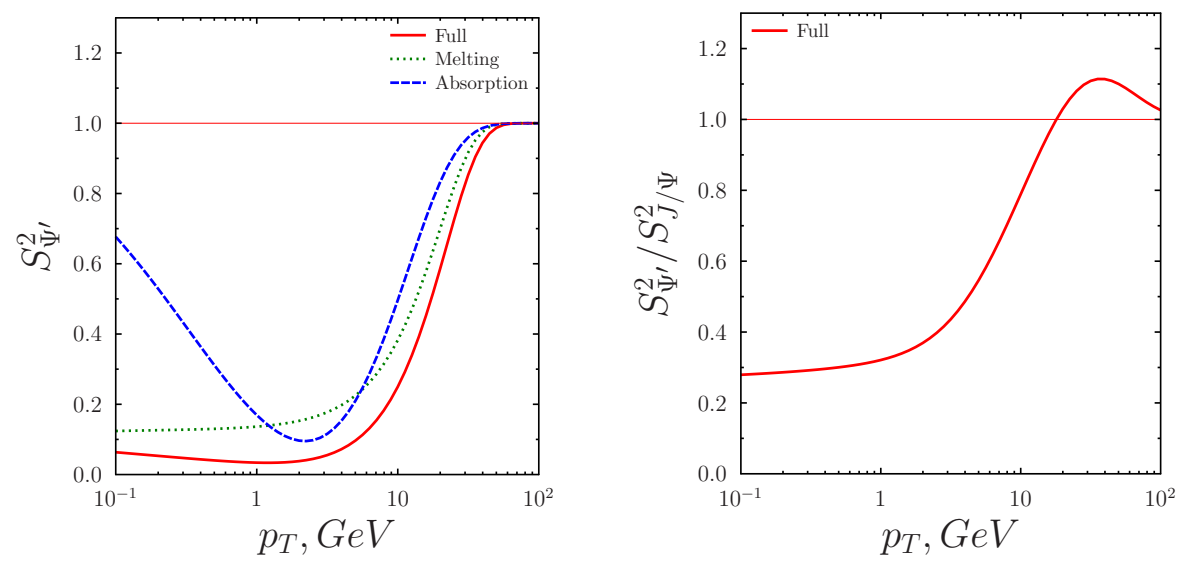

Figure 5. Left: The same as in Fig. 4(right), but for production of a $\psi(2 S)$ radial excitation. Right: Ratio of production rates of $\psi(2 S)$ to $J / \psi$ calculated for central lead-lead collisions with $q_{0}=2 \mathrm{GeV}^{2} / \mathrm{fm}$. 
strates a peculiar feature: at large $p_{\psi} \gtrsim 10 \mathrm{GeV}$. It exceeds unity, at variance with the naively expected stronger in-medium attenuation of $\psi(2 S)$. This is related to the effect of nuclear enhancement of the $\psi(2 S)$ photo-production rate, predicted in $[6,27]$. This effect is related to the specific structure of the $\psi(2 S)$ wave function $\Psi_{\psi}(r)$, which has a node and changes sign at $r \approx 0.4 \mathrm{fm}$ (see Fig. 4 in [16]). This interesting effect is expected to occur at sufficiently high $p_{\psi}$.

\subsection{Azimuthal asymmetry}

A strong charmonium suppression FSI interactions unavoidably leads to an azimuthal asymmetry, characterised by the parameter $v_{2}=\langle\cos (2 \phi)\rangle$, where $\phi$ defined in (18), is the azimuthal angle between the $J / \psi$ trajectory and the scattering plane. Indeed, a charmonia propagated along the long or short diameters of the nuclear intersection area, cover different path lengths in the medium, so they are suppressed differently, which gives rise to an azimuthal asymmetry. Correspondingly, we can calculate $v_{2}(b)$ as,

$$
\begin{aligned}
v_{2}(b) & =\int_{0}^{2 \pi} \frac{d \phi}{2 \pi} \cos (2 \phi) \int d^{2} s \frac{d^{2} s T_{A}(\vec{s}) T_{B}(\vec{b}-\vec{s})}{T_{A B}(b)\left|S_{J / \psi}(b)\right|^{2}} \\
& \times\left|\frac{\int d^{2} r_{1} d^{2} r_{2} d z_{1}^{-} d z_{2}^{-} \Psi_{f}^{\dagger}\left(\vec{r}_{2}, z_{2}^{-}\right) G\left(\infty, \vec{r}_{2}, z_{2}^{-} ; 0, \vec{r}_{1}, z_{1}^{-}\right) \Psi_{i n}\left(\vec{r}_{1}, z_{1}^{-}\right)}{\int d^{2} r d z^{-} \Psi_{f}^{\dagger}\left(\vec{r}, z^{-}\right) \Psi_{i n}\left(\vec{r}, z^{-}\right)}\right|^{2}
\end{aligned}
$$

The results obtained with $q_{0}=2 \mathrm{GeV}^{2} / \mathrm{fm}$, are plotted in Fig. 6 as function of $J / \psi$ momentum for several centralities. Naturally, $v_{2}$ vanishes at very large momenta, where the medium becomes fully

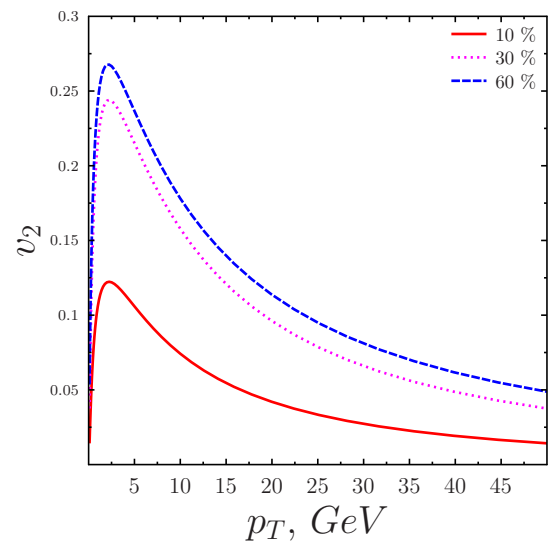

Figure 6. Azimuthal asymmetry parameter $v_{2}$ for $J / \psi$ production in lead-lead collisions calculated with $q_{0}=$ $2 \mathrm{GeV}^{2} / \mathrm{fm}$. The curves represent different centralities, from bottom to top $10 \%, 30 \%$ and $60 \%$.

transparent, as was demonstrated if Fig. 4. The parameter $v_{2}$ at intermediate and large momenta has a magnitude similar to what was measured at LHC and calculated in [26] for light hadrons. It is also close to what was observed for $J / \psi$ by the CMS experiment recently [28]. It is worth emphasizing that the azimuthal asymmetry is not affected by ISI, because the expected effects [29] are very weak. Therefore, the calculated $v_{2}$ could be directly compared with data, provided that we did not miss any 
significant FSI effect. That, however, does not seem to be the case, recent data from LHC give a strong indication for presence of a mechanism enhancing $J / \psi$, at least at low $p_{T}$ (see below), so we restrain here from a direct comparison with data.

\subsection{Regeneration of charmonia}

One cannot treat the ISI stage as production of a charmonium, but of a small-size $\bar{c} c$. The charmonium wave function is formed afterwards within the second stage of FSI. The production time of a $\bar{c} c$ pair in the c.m. of the collision of two nuclei is $t_{c}=\left(4 m_{c}^{2}+p_{T}^{2}\right)^{-1 / 2}$. This time is much shorter than the formation time of the charmonium wave function, $t_{f}=E_{T} / 2 \omega$. There are two options for the $\bar{c} c$, which emerges from the ISI stage: the pair is either colour singlet $(\mathrm{S})$, or octet $(\mathrm{O})$, and the latter dominates. Indeed, the corresponding cross sections read,

$$
\begin{aligned}
\sigma\left(g g \rightarrow\{\bar{c} c\}_{1}\right) & =\frac{1}{8} \int d^{2} r K_{0}\left(m_{c} r\right) \sigma_{\bar{c} c}(r), \\
\sigma\left(g g \rightarrow\{\bar{c} c\}_{8}\right) & =\int d^{2} r K_{0}\left(m_{c} r\right) \sigma_{g \bar{c} c}(r),
\end{aligned}
$$

where $K_{0}$ is the modified Bessel function;

$$
\sigma_{g \bar{c} c}\left(r_{T}\right)=\frac{9}{4} \sigma_{\bar{c} c}\left(r_{T} / 2\right)-\frac{1}{8} \sigma_{\bar{c} c}\left(r_{T}\right) \approx \frac{7}{16} C\left(E_{\bar{c} c}\right) r_{T}^{2} .
$$

From this comparison we conclude that the production rate of a colour octet $\bar{c} c 3.5$ times exceeds that for a colour singlet.

Further multiple interactions in the dense medium can convert the initially produced octet to a singlet, and thus enhance charmonium production. The evolution of a $\bar{c} c$ pair, initially either a singlet, or an octet, in the medium can be described by the following equations,

$$
\begin{aligned}
& W(S \rightarrow S)=\frac{1}{9}+\frac{8}{9} \exp \left[-\frac{9}{32} v_{\psi} \int_{0}^{L} d l r^{2}(l) \hat{q}(l)\right] W_{0}(S) \\
& W(O \rightarrow S)=\frac{1}{9}-\frac{1}{9} \exp \left[-\frac{9}{32} v_{\psi} \int_{0}^{L} d l r^{2}(l) \hat{q}(l)\right] W_{0}(O),
\end{aligned}
$$

Notice, that even in a very dense medium, with a very large number of multiple dipole interactions, the probability of final production of a colour singlet, does not vanish, but saturates at $W(O)=1 / N_{c}^{2}=$ 1/9. It is clear why: since a quark has three colour components, a $\bar{q} q$ pair has 9 components, equaly populated after many colour exchanges. Only one of them is a colour singlet.

Since the mean dipole separation $r^{2}(l)$ rises with $l$, the probability of the regeneration processes Eq. (24) is expected to be strongest at smaller $p_{T}$ of the charmonium. At large $p_{T}$ the Lorentz time dilation "freezes" the initial small size of the dipole and the probability of regeneration fades out.

The solution of the set of equations, analogous to (23)-(24), but based on the path-integral technique, will be published elsewhere [30].

\section{Summary}

The main objective of this paper is improvement available theoretical tools for evaluation of the effect of Debye screening of the charmonium binding potential in a hot medium, and to draw attention to the 
importance of the frequently forgotten alternative mechanism of charmonium attenuation, absorption. Our major observations are as follows.

We demonstrated that even in the limit of infinitely high temperature, when Debye screening completely eliminates the binding potential, the survival probability of a charmonium is not zero. Moreover, it approaches unity at large transverse momenta of the charmonium.

We developed a procedure of Lorentz boosting of the binding potential and screening corrections. In the boosted Schrödinger equation the real part of the potential is directly related to the rest frame binding potential.

Another source of charmonium attenuation in the medium is the possibility of colour-exchange interactions of the $\bar{c} c$ pair with the medium. Such an interaction, usually called absorption, contributes to the imaginary part of the potential. The effect of absorption is subject to colour transparency, therefore it vanishes at large charmonium momenta.

Solving equation for the Green function with a realistic model for the coordinate and time dependences of the temperature and transport coefficient, we calculated the suppression factor for a $J / \psi$ produced in central lead-lead collisions including both melting and absorption. We concluded that these mechanisms give comparable contributions to the $J / \psi$ suppression. Similar calculations are performed for the radial excitation $\psi(2 S)$, which found to be stronger suppressed, except at very large transverse momenta.

Attenuation of a charmonium produced in nuclear collisions unavoidably leads to an azimuthal asymmetry for non-central collisions. We calculated the parameter $v_{2}$ and found it to have a magnitude comparable with data.

A novel mechanism of regeneration of colourless $\bar{c} c$ dipoles by multiple interactions in a dense medium is proposed.

Acknowledgments: A partial support by Fondecyt (Chile) grants 1130543, 1130549, 1140390, and 1140377 is kindly acknowledged.

\section{References}

[1] B. Abelev et al. [ALICE Collaboration], Phys. Lett. B 718, 295 (2012) [arXiv:1203.3641 [hepex]].

[2] F. Karsch, D. Kharzeev and H. Satz, Phys. Lett. B 637, 75 (2006) [hep-ph/0512239].

[3] M. Laine, O. Philipsen, P. Romatschke, M. Tassler; JHEP 0703, 054 (2007); [arXiv: hep$\mathrm{ph} / 0611300]$

[4] B. Z. Kopeliovich, I. K. Potashnikova, I. Schmidt and M. Siddikov, arXiv:1407.8080 [nucl-th]; to appear in Nucl. Phys. A.

[5] B. Z. Kopeliovich, I. K. Potashnikova and I. Schmidt, Phys. Rev. C 82, 024901 (2010) [arXiv:1006.3042 [nucl-th]].

[6] B. Z. Kopeliovich and B. G. Zakharov, Phys. Rev. D 44, 3466 (1991).

[7] B. Z. Kopeliovich and B. G. Zakharov, Phys. Lett. B 264, 434 (1991).

[8] R. J. Glauber, in Lectures in Theoretical Physics, W. E. Brittin et al Editors, New York (1959).

[9] B. Z. Kopeliovich, L. I. Lapidus and A. B. Zamolodchikov, JETP Lett. 33, 595 (1981) [Pisma Zh. Eksp. Teor. Fiz. 33, 612 (1981)].

[10] R. Baier, Y. L. Dokshitzer, S. Peigne and D. Schiff, Phys. Lett. B 345, 277 (1995); Nucl. Phys. B 484, 265 (1997).

[11] M. B. Johnson, B. Z. Kopeliovich and A. V. Tarasov, Phys. Rev. C 63, 035203 (2001) [hep$\mathrm{ph} / 0006326]$. 
[12] B. Z. Kopeliovich, I. K. Potashnikova and I. Schmidt, Phys. Rev. C 81, 035204 (2010) [arXiv:1001.4281 [hep-ph]].

[13] V. N. Gribov, Sov. Phys. JETP 29, 483 (1969); Zh. Eksp. Teor. Fiz. 56, 892 (1969).

[14] B. Z. Kopeliovich, A. Schafer and A. V. Tarasov, Phys. Rev. D 62, 054022 (2000) [hep$\mathrm{ph} / 9908245]$.

[15] B. Z. Kopeliovich, A. V. Tarasov and A. Schäfer, Phys. Rev. C 59, 1609 (1999) [hep$\mathrm{ph} / 9808378]$.

[16] J. Hüfner, Y. .P. Ivanov, B. Z. Kopeliovich and A. V. Tarasov, Phys. Rev. D 62, 094022 (2000) [hep-ph/0007111].

[17] A. Bazavov and P. Petreczky, Nucl. Phys. A904-905 2013, 599c (2013); J. Phys. Conf. Ser. 458, 012012 (2013); J. Phys. Conf. Ser. 432, 012003 (2013).

[18] O. Kaczmarek, S. Ejiri, F. Karsch, E. Laermann and F. Zantow, Prog. Theor. Phys. Suppl. 153, 287 (2004) [hep-lat/0312015].

[19] O. Kaczmarek, “Recent Developments in Lattice Studies for Quarkonia,” arXiv:1208.4075 [heplat].

[20] J. G. Korner and G. Thompson, Phys. Lett. B 264, 185 (1991).

[21] B. Kopeliovich, A. Tarasov and J. Hüfner, Nucl. Phys. A 696, 669 (2001) [hep-ph/0104256].

[22] B. Z. Kopeliovich, E. Levin, Ivan Schmidt and M. Siddikov, paper in preparation.

[23] X.-F. Chen, C. Greiner , E. Wang , X.-N. Wang, Z. Xu, Phys.Rev. C81 (2010) 064908 [arXiv:1002.1165 [nucl-th]].

[24] E. Eichten, K. Gottfried, T. Konoshita, K. D. Lane, and T.-M. Yan, Phys. Rev. D 17, 3090 (1978); 21, 203 (1980).

[25] F. Karsch, M. T. Mehr and H. Satz, Z. Phys. C 37, 617 (1988).

[26] B. Z. Kopeliovich, J. Nemchik, I. K. Potashnikova and I. Schmidt, Phys. Rev. C 86, 054904 (2012) [arXiv:1208.4951 [hep-ph]].

[27] B. Z. Kopeliovich, J. Nemchick, N. N. Nikolaev and B. G. Zakharov, Phys. Lett. B 309, 179 (1993) [hep-ph/9305225].

[28] Dong Ho Moon (for the CMS Collaboration), a talk at Quark Matter 2014, Darmshtadt, May 19-24, 2014.

[29] B. Z. Kopeliovich, A. H. Rezaeian and I. Schmidt, Phys. Rev. D 78, 114009 (2008) [arXiv:0809.4327 [hep-ph]].

[30] B. Z. Kopeliovich, I. K. Potashnikova, I. Schmidt, M. Siddikov, work in progress. 